\title{
An Agent Based Model for the Simulation of Transport Demand and Land Use
}

\author{
Nam Huynh, Vu Lam Cao, Rohan Wickramasuriya, Matthew Berryman, Pascal Perez and Johan Barthelemy \\ SMART Infrastructure Facility, University of Wollongong, NSW, Australia
}

\begin{abstract}
Agent based modelling has emerged as a promising tool to provide planners with insights on social behaviour and the interdependencies characterising urban system, particularly with respect to transport and infrastructure planning. This paper presents an agent based model for the simulation of land use and transport demand of an urban area of Sydney, Australia. Each individual in the model has a travel diary which comprises a sequence of trips the person makes in a representative day as well as trip attributes such as travel mode, trip purpose, and departure time. Individuals are associated with each other by their household relationship, which helps define the interdependencies of their travel diary and constrains their mode choice. This allows the model to not only realistically reproduce how the current population uses existing transport infrastructure but more importantly provide comprehensive insight into future transport demands. The router of the traffic micro-simulator TRANSIMS is incorporated in the model to inform the actual travel time of each trip and changes of traffic density on the road network. Simulation results show very good agreement with survey data in terms of the distribution of trips done by transport modes and by trip purposes, as well as the traffic density along the main road in the study area.
\end{abstract}

Keywords: Agent Based Model, TRANSIMS, Road Traffic, Transport Demand, Urban Planning

\section{INTRODUCTION}

The ability to realistically predict the demand of transport and traffic on the road network is of critical importance to efficient urban transport and infrastructure planning. Agent based models of urban planning have been increasingly introduced over the last decades. Many agent based models for transport and urban planning can be found in the literature with different geographical scales and at various levels of complexity of agent's behaviours and autonomy. They proved that with a large real world scenario, agent based modelling, while being able to reproduce the complexity of an urban area and predict emergent behaviours in the area, has no issue with the performance ${ }^{1}$. They also show that for traffic and transport simulation purposes, agent based modelling has been considered as a reliable and well worth developing tool that planners can employ to build and evaluate alternative scenarios of an urban area.

Many models that have been reported in the literature however are unable to explicitly simulate the dynamic interactions between the population growth, the transport/traffic demands, urban mobility (i.e. residential relocation of households), and the resulting changes in how the population perceive the liveability of an urban area. The agent based model presented in this paper aims at addressing this gap in the literature. The heterogeneity of the population is represented in the model in terms of demographic characteristics, environmental perception, and decision making behaviour. Inherently, the simulated

1 Vogel, A. and Nagel, K. Multi-agent based simulation of individual traffic in Berlin. CUPUM 2005 Conference, http://128.40.111.250/ cupum/searchpapers/papers/ paper179.pdf (2005) 
population will evolve over time facilitating the interactions between dynamics of residential relocation of households, transportation behaviours and population growth. Thanks to this feature, the model can be used for exploring long-term (e.g. 20 year time horizon) consequences of various transport and land use planning scenarios.

Individuals are represented in this model as autonomous decision makers that make decisions that affect their environment (i.e. travel mode choice and relocation choice) as well as are required to make decisions in reaction to changes in their environment (e.g. family situation, employment). With respect to transportation, each individual has a travel diary which comprises a sequence of trips the person makes in a representative day as well as trip attributes such as travel mode, trip purpose, and departure time. Individuals in the model are associated with each other by their household relationship, which helps define the interdependencies of their travel diary and constrains their mode choice. This feature, together with the interactions between urban mobility, transportation behaviours, and population growth, allows the model to not only realistically reproduce how the current population uses existing transport infrastructure but more importantly provide comprehensive insight into its future transport demands. The router of the traffic micro-simulation package TRANSIMS is incorporated in the agent based model to inform the actual travel time of each trip (which agents use in considering new travel modes) and changes of traffic density on the road network.

Major components that constitute the agent based model in this study are (i) synthetic population, (ii) residential relocation choice, (iii) perceived liveability, (iv) travel diaries, (v) traffic micro-simulation, and (vi) transport mode choice. These components equip the model with unique features that allows it to be used as a comprehensive tool for assisting integrated travel - land use planning. These components are briefly described in Section 2 in order to provide a full picture of the model features and capabilities. The focus of this work however will be in reporting the simulation results in regards to road traffic and transport demands (Section 3). The paper closes with discussions on further developments of the model.

\section{MODEL COMPONENTS}

This section provides an overview of the six main components that constitute the agent based model in this study².

\section{Synthetic population}

The purpose of the synthetic population is to create a valid computational representation of the population in the study area that matches the distribution of individuals and household as per the demographics from census data. The construction of the synthetic population involves the creation of a proto-population calibrated on socio-demographic information provided by the Australian census data (full enumeration). Different to the majority of existing algorithms for constructing a synthetic population, the algorithm used in this study uses only aggregated data of demographic distributions as inputs, i.e. no disaggregated records of individuals or households (e.g. a survey) are required ${ }^{3}$. The resulting synthetic population is made of individuals belonging to specific households and associated with each other by household relationship.

This initial population is evolved according to annual increments during the simulation period. Each individual and household is susceptible to various demographic (e.g. aging, coupling, divorcing, reproducing of individuals) and economic changes controlled by conditional probabilities. The consequent changes in the structure of households as a result of these processes are also captured. An immigrant population may be added to the existent population of the study area at the end of each simulation step.

\section{Residential location choice}

Household relocation modelling is an integral part of both the residential and transport planning processes as household locations determine demand for community facilities and services, including transport network demands. The approach used to model residential location choice includes two distinct processes ${ }^{4}$ : the decision to relocate, and the process of finding a new dwelling. A multinomial logit model was used to represent the process by which households make

2 Berryman, M. J., Denamagage, R. W., Cao, V. \& Perez, P. Modelling and data frameworks for understanding infrastructure systems throuh a systems-of-systems lens. International Symposium for Next Generation Infrastructure 2013, 1-13, University of Wollongong, Wollongong (2013).

3 Huynh, N., Namazi-Rad, M., Perez, P., Berryman, M. J., Chen, Q. \& Barthelemy, J. Generating a synthetic population in support of agent-based modeling of transportation. Sydney 20th International Congress on Modelling and Simulation, 1357-1363, The Modelling and Simulation Society of Australia and New Zealand, Australia (2013).

4 Namazi Rad, M., Shukla, N., Munoz Aneiros, A., Mokhtarian Dehkordi, P. \& Ma, J. A probabilistic predictive model for residual mobility in Australia. International Symposium for Next Generation Infrastructure 2013, 1-6, University of Wollongong, Wollongong (2013). 
decision to relocate. The attributes of this model are change in household income, change of household configuration (e.g. having a newborn, divorced couples, newly wed couples), and the tenure of the household. The HILDA data was used to regress the coefficients associated to each of these attributes needed in the binomial logit model.

Once a household is selected for relocation, the second decision determines where the household will relocate and whether they will be renting or buying a dwelling in the target location, if a suitable a dwelling is found. This process of finding a new dwelling is modelled as a constraint satisfaction process, whereby each household will attempt to find a suitable dwelling based on three factors, affordability, availability, and satisfaction.

\section{Perceived liveability}

A significant departure of the current model to other existing approaches is the assumption that residential location choice is based not only on availability and affordability principles but also on the perception that individuals have of the quality of their living environment. The perceived liveability component uses a semi-empirical model to estimate individual levels of attraction to and satisfaction with specific locations $s^{5,6}$.The semi-empirical model is a statistical weighted linear model calibrated on a computer assisted telephone interviewing survey data collected in the study area.

\section{Travel diaries}

Each individual in the synthetic population is assigned with a travel diary which comprises a sequence of trips the person makes in a representative day as well as trip attributes such as travel mode, trip purpose, departure time, origin and destination. Because these details of travel behaviours of the population are not completely available in any single source of data (for confidentiality reasons), the process of assigning travel diaries to individuals comprises two steps. The first step assigns a trip sequence each individual makes in a representative day using the Household Travel Survey data. Details of each trip in this trip sequence include trip purpose, travel mode, and departure time. The second step assigns locations to the origin and destination of each trip in the trip sequence.

Due to changes in the synthetic household attributes (e.g. household type, number of children under 15, etc) as the population evolves, travel diaries may need to be reassigned in subsequent simulation steps to these households in the model.

\section{Traffic micro simulation}

TRANSIMS was chosen as the traffic micro-simulator as, in its current iteration, it is a clean, efficient, C++-based platform that supports an individual level of modelling.

Normally one would use a process analogous to simulated annealing to arrive at the solution; running the router to establish initial routes, then finding when vehicles jam, and either redirecting them off the street temporarily into a park (if the numbers are sufficiently low) or by then re-routing them using the router and then running the simulation until numbers jammed are sufficiently low. Given the typical travel volumes (around 100,000 commuters), and our desire to simulate a 20-year period, we are forced to run only one typical weekday and weekend in simulation per year, and run only one iteration of the router. We have compared this with test runs of multiple iterations of router and the core microsimulator of vehicle movements, and found that travel times are within 5\%; this we consider sufficient for our purposes.

\section{Transport mode choice}

The purpose of the travel mode choice algorithm was to accurately describe the decision-making processes of individuals travelling on the transport network in the study area, thus enabling the prediction of the choice of travel modes of individuals. Travel modes considered in this study are car driver, car passenger, public transport, taxi, bicycle, walk, and other.

A multinomial logit (MNL) model was developed for this purpose. At the heart of the MNL formulation is a linear part-worth utility function that calculates the utility of each alternative travel mode choice. Independent variables for this function include the difference of fixed cost and difference of variable cost of the selected travel mode with the cheapest mode. The variable cost is dependent on the estimated travel time, which is the output of the traffic micro-

5 Namazi Rad, M., Perez, P., Berryman, M. \& Lamy, F. An experimental determination of perceived liveability in Sydney. ACSPRI Conferences, RC33 Eighth International Conference on Social Science Methodology, 1-13, ACSPRI, Australia: (2012).

6 Namazi Rad, M., Lamy, F., Perez, P. \& Berryman, M. A heuristic analytical technique for location-based liveability measurement. The Fifth Annual ASEARC Research Conference: Looking to the future, 35-38, University of Wollongong, Wollongong (2012). 
simulation. Another independent variable is the individual's income, acting as a proxy for the individual's perception of value of time. MNL regression was used on the HTS data to estimate the utility coefficients.

\section{Simulation results with regards to transport demands and road traffic}

The agent based model described in Section 2 is applied to simulate the dynamic interactions between population growth, urban relocation choice and transport demands for Randwick - Green Square, a metropolitan area in south east of Sydney, Australia. This area has a population of approximately 110000 individuals in around 52000 households that live in private dwellings.

The simulation period is from 2006 to 2011 . The initial synthetic population is constructed using the 2006 census data that is available from the Australian Bureau of Statistics. This initial synthetic population was validated that it matches the demographics of the real population at both individual level and household level, and thus is a realistic computational representation of the real population in the area ${ }^{3}$. It was also shown that the synthetic population in year 2011 (i.e. after 5 simulation years) matches the demographics of the population in the study area as described in the 2011 census data. This affirmed that the algorithm to evolve the population while simulating the evolution at individual level can capture the dynamics of household structures in the population.

Figures 1 and 2 respectively show the percentage of trips by each mode and each purpose with respect to the total number of trips made by the whole population for year 2006 (initial year) and simulation year 2011. Figure 3 compares the percentage of individuals in the synthetic population against that in the HTS data by the number of trips made daily. The distributions in these graphs are in very good agreement with the HTS data for the whole Sydney Greater Metropolitan Area.

Trip counts by purposes over 24 hours of a representative day in year 2011 are shown in Figure 4. It can be observed that the model can realistically reproduce the patterns of travel demand of the population in the study area as well as the change of these patterns as the population evolves.

Traffic density (that was outputted from TRANSIMS) at one major intersection along Anzac Parade, the main road in the study area, in the morning peak hour (8.00am to 9.00am) compared against its congestion profile from Google Maps ${ }^{7}$ is shown in Figure 5. The model is able to reproduce the relatively higher northbound traffic density. These results are in agreement with observations of traffic profiles from Google Maps. Qualitative trends of traffic counts at major cross sections from simulation results were also analysed and in good agreement with survey data in the study area.

7 Google Maps, https://maps.google.com/ (accessed on 31/01/2014) 


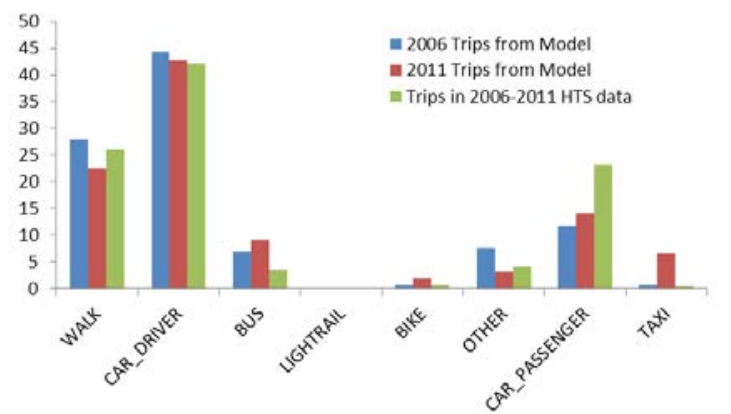

Figure 1: Percentage of trips by modes from simulation years 2006 and 2011 vs 2006-2011 HTS data.

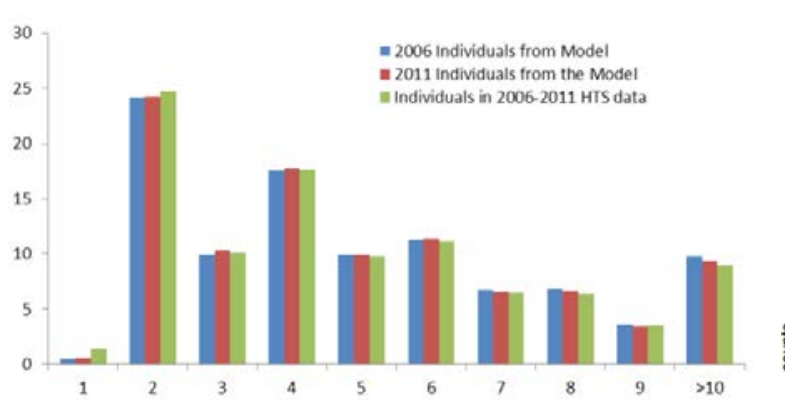

Figure 3: Percentage of population by number of daily trips for simulation years 2006 and 2011 vs 2006-2011 HTS data.

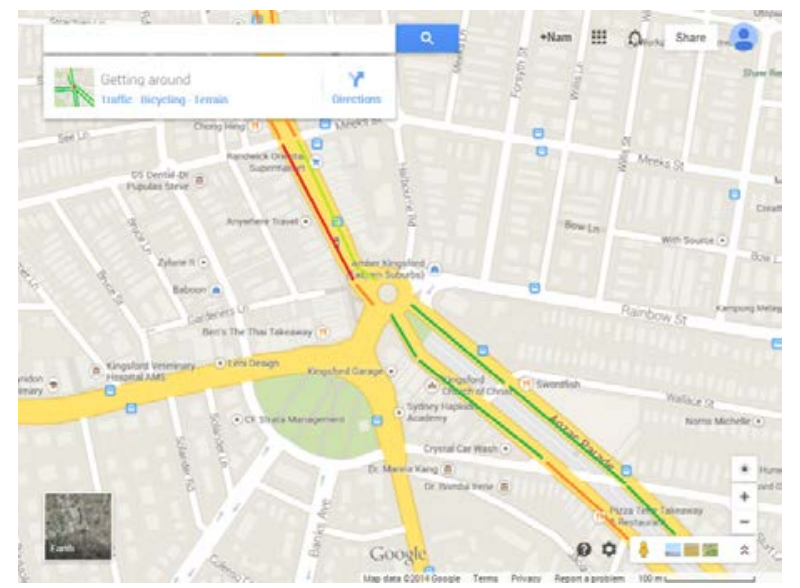

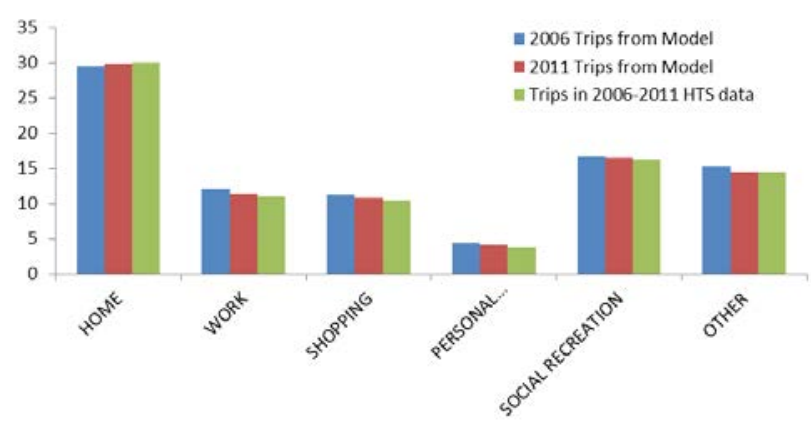

Figure 2: Percentage of trips by purposes from simulation years 2006 and 2011 vs 2006-2011 HTS data.

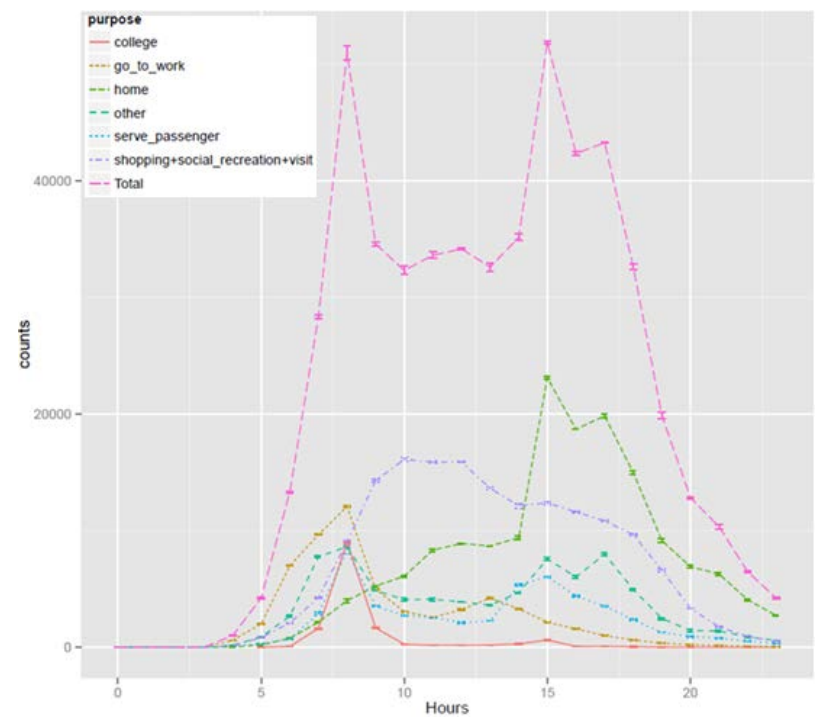

Figure 4: Trip counts by purposes over 24 hours of a representative day in year 2011.

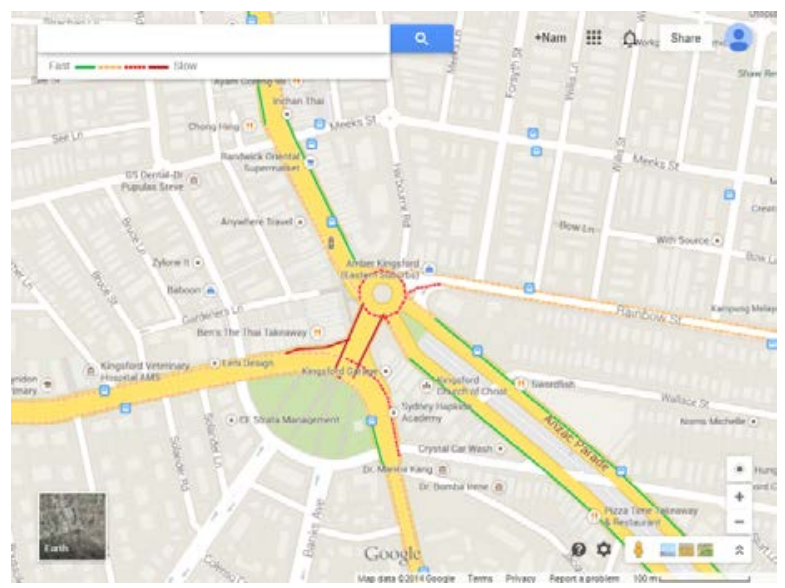

Figure 5: Traffic density on Anzac Parade near the intersection with Rainbow street in the morning peak hour. Left panel: traffic density from simulation results. Right panel: congestion profile from Google Maps 
Such agreement however does not occur on all parts of the road network. This could be attributed to the randomness in the assignment of activity locations to origin and destination of trips in the travel diaries of the population. While the assignment of destination locations of trips related to work is constrained by the Journey To Work data, the randomness in assigning destination locations to trips of other purposes does not guarantee a realistic representation of traffic profiles in the model.

\section{CONCLUSIONS}

This paper has presented an agent based model for the simulation of transport demands and land use for an urban area in south east Sydney, Australia. Being comprised of six major components the model is able to capture the decision making of the population with respect to relocation and transport, and thus is able to explicitly simulate the dynamic interactions between population growth, transport demands, and urban land use. This is a unique feature that has not been found in many other agent based models for urban transport and urban planning. Thanks to this feature, the model can be used for exploring long-term consequences of various transport and land use planning scenarios.

Various aspects of the simulation results on transport demands of the study area were presented. Being in good agreement with the corresponding survey data, these results affirm that the model's capability to realistically reproduce travel demand of an urban area and any changes to this travel demand as the population evolves. This is because individuals in the model are associated with each other by their household relationship, which helps define the interdependencies of their travel diary and constrains their mode choice.

Traffic density at various locations along the main road in the study area also matches with the observations of traffic congestion on the same road from Google Maps. Mismatches however occur on other (smaller) roads in the study area. This could be attributed to two factors. The first is the lack of a survey data on the origin and destination of non-work trips. The randomness in assigning a location to the destinations of these trips obviously cannot guarantee a realistic representation of traffic demands in the simulation model. The second factor is the limited ability of the TRANSIMS router to realistically reproduce the reasoning of a person in choosing a possible route for the trips the person makes, including dynamic routing to avoid heavy traffic in real time. 